\title{
Occupational stress and job burnout in mental health
}

\section{Stress lavorativo e burnout in salute mentale}

\author{
A. Lasalvia*, Guest Editor and M. Tansella, Editor
}

Professionals who care for long-term and seriously ill patients frequently experience distressing emotional situations and profound suffering. These emotions can include the need to 'rescue' patients, a sense of failure and frustration when the illness progresses, feelings of powerlessness against the illness and its associated losses, grief, fear of becoming ill oneself and a desire to remain emotionally distant from and avoid patients to escape from these feelings. Although they are common in everyday clinical practice, such emotions often affect the quality of medical care that physicians provide and their well-being (Meier et al., 2001). Individuals who work in the mental health field have been identified as a high-risk group for occupational stress (Leary \& Brown, 1995; Nolan et al., 1995) and burnout (Leiter \& Harvie, 1996; Prosser et al., 1996; Thomsen et al., 1999).

Burnout should be distinguished conceptually from occupational stress, which is a generic term that refers to temporary adaptation at work, accompanied by mental and physical symptoms. In contrast, burnout is considered to be the final stage in a breakdown during adaptation and therefore results from prolonged occupational stress. Moreover, burnout includes the development of dysfunctional attitudes and behaviours towards the recipients of one's care or services and towards one's job and organisation.

Burnout is a critical issue for mental health-care delivery, because it can lead to decreased work performance and, ultimately, poorer treatment outcomes (Priebe et al., 2004). Mental health services throughout Europe are facing severe financial shortages and consequent shortfalls in the number of sector-employed professionals. The situation is particularly exigent in European countries with a national health service, such as the UK (Sainsbury Centre for Mental Health, 2001) and Italy (Lasalvia et al., 2007). Thus, workers' feelings of job disaffection and burnout can thin a mental health service workforce.

* Address for correspondence: Dr Antonio Lasalvia, Department of Public Health and Community Medicine, Section of Psychiatry and Clinical Psychology, Ospedale Policlinico 'G.B. Rossi', Piazzale L.A. Scuro 10, 37134 Verona, Italy.

(Email: antonio.lasalvia@univr.it)
Historically, burnout has been considered more of a personal problem than an organisational one (Maslach \& Leiter, 1997). Studies that explored determinants of burnout in mental health settings generally focused on individual socio-demographic factors (Fagin et al., 1996; Edwards et al., 2000), individual occupational characteristics (Prosser et al., 1999; Kilfedder et al., 2001; Evans et al., 2006) and predisposing personality traits (Naisberg-Fennig et al., 1991; Deary et al., 1996a). Yet, recent research has expanded the theoretical burnout framework to include perceived organisational sources of stress. Maslach \& Leiter (1997) proposed that burnout develops as a result of mismatches between professionals and their job contexts in several worklife areas (i.e. workload, control, rewards, community, fairness and values). Specifically, a discrepancy is perceived when the process of establishing a psychological 'contract' with one's job leaves critical issues unresolved or when changes in working relationships feel unacceptable to the worker. Accordingly, Leiter \& Maslach (2000) proposed the 'Mediation Model', which postulates that the greater the degree of workerjob mismatch, the greater the likelihood of burnout. The Mediation Model can be used in research and applied intervention, because it focuses on the relationship between burnout and contextual work sources (Leiter \& Maslach, 2004). Thus, burnout is viewed as an individual syndrome that develops in a work context, where perceived organisational factors have greater influence than personal factors (Maslach \& Leiter, 2008).

A recent, large-scale survey that examined Italian mental health staff (Lasalvia et al., 2009), adopting the framework of Leiter \& Maslach's Mediation Model found that perceived organisational factors were the only significant predictors of the three dimensions of burnout (emotional exhaustion, cynicism and professional efficacy). Moreover, specific predictors accounted for each dimension, with no significant professional category effects. With regard to strength of association, high workload was most predictive of exhaustion; poor rewards and few perceived positive service changes were the best predictors of disengagement from work; and lack of participation in decision-making was most predictive of reduced professional efficacy. These findings are consistent with the 'Job Demand-Resources' (JD-R) 
model, which postulates that job demands and lack of job resources are linked specifically to emotional exhaustion and depersonalisation, respectively (Bakker \& Demerouti, 2007). The model assumes that two underlying processes mediate the development of burnout: (a) energy depletion is driven by high job demands (e.g. time pressure, emotional demands, cognitive demands and shift work) and is associated with exhaustion and (b) erosion of motivation is driven by a lack of job resources (e.g. supervisor support, feedback, control, task variety and financial rewards) and is associated with disengagement (cynicism) and a lack of efficacy.

This issue of Epidemiology and Psychiatric Sciences contains three Editorials that discuss the pressing issue of occupational stress and job burnout among mental health staff. We invited three of the world's prominent experts who conduct empirical research in this field.

Dollard \& McTernan (2011) address overall conceptual issues, proposing a new construct, the Psychosocial Safety Climate (PSC), to better understand the interpersonal and psychological dynamics that occur within organisations. PSC has been defined as shared perceptions of organisational policies, practices and procedures for the protection of workers' psychological health and safety that stem largely from management practices. The concept of PSC is derived from the notion that PSC flows principally from the priority that is given by senior management to production $v$. the psychological health of workers - where there is political will and when senior management favours a balance, several policies, practices and procedures that reflect this process are expected (Dollard \& Karasek, 2010). Based on this principle, working conditions (i.e. job demands and resources) derive from the initiatives of senior managers.

PSC theory extends the JD-R model. Thus, PSC is viewed as a precursor or antecedent to working conditions - not an outcome or mediator. Recent studies have demonstrated that PSC is clearly an antecedent to the work context and is associated with psychological health through its relationship with the working environment (Bond et al., 2010; Dollard \& Bakker, 2010). In fact, PSC correlates negatively with job demands and is positively associated with job resources. Job demands, in turn, predict burnout (i.e. exhaustion and cynicism), whereas job resources predict engagement. Both burnout and engagement are associated with performance. Thus, PSC affects job performance via health erosion (i.e. burnout) and motivational pathways (i.e. work engagement). Considering the characteristics of PSC, an organisation that strongly advocates employee psychological health and safety will create job demands that are motivating and conducive to health (Dollard \& Bakker, 2010). PSC is therefore a lead indicator of workplace psychosocial hazards (high demands and low resources), psychological health and employee engagement and a potential moderator of psychosocial hazard effects. Consequently, PSC can be viewed as an efficient target for improving working conditions and, in turn, reducing burnout and increasing engagement and productivity.

Among mental health staff, psychiatrists are at high risk of depression, anxiety, psychiatric caseness and burnout (Naisberg-Fennig et al., 1991; Deary et al., 1996b; Thomsen et al., 1999; Lasalvia et al., 2009). In the second editorial, Kumar (2011) focuses on occupational stress and job burnout in psychiatrists. Factors that render psychiatry a stressful profession are examined first (with a specific emphasis on occupational stressors), such as patient violence and suicide, limited resources, crowded inpatient wards, changing cultures in mental health services, high work demands, poorly defined roles of consultants, responsibility without authority, inability to effect systemic change, conflict between responsibility towards employers $v$. the patient and isolation. Mechanisms that lead to burnout among psychiatrists are then considered, and the negative outcomes of chronic exposure to external job-related stressors (i.e. professional burnout) are discussed. Finally, while acknowledging that many advances have been made in our comprehension of the factors that are associated with stress and burnout in psychiatrists, Kumar (2011) highlights the need to gain a greater understanding of the determinants of positive work environments and the factors that protect psychiatrists against work stress, calling for a proper examination of the parameters that help psychiatrists find enjoyment or a sense of purpose in their work.

In the third editorial, Priebe \& Reininghaus (2011) challenge the traditional research paradigm, which focuses on stress and burnout, and encourage a shift from the negative stress model to an approach that emphasises the positive sides of work - i.e. the factors that promote 'work engagement', a positive, fulfilling, effective-motivational state of work-related well-being that can be viewed as the antipode of job burnout (Bakker et al., 2008). The overall conceptual framework of this shift is 'positive psychology' (Seligman \& Csikszentmihalyi, 2000), a new research and application field that describes aspects of the human condition that lead to happiness and fulfilment to determine the factors that better one's life (rather than trying to prevent negative situations) (Caprara et al., 2010). Thus, the concept of work engagement can help capture aspects that contribute to positive attitudes towards work and determine organisational 
well-being. A profession in mental health generally provides not only a wide range of experiences, relationships and encounters with risks for frustration and disappointment but also joy, fascination and satisfaction - it is no wonder that positive relationships with patients and the challenges of the work have been reported the most enjoyable aspects of the occupation (Prosser et al., 1996; Galeazzi et al., 2004). The authors, therefore, urge future research to investigate the rewards of working in mental health and the factors that make it a fascinating and challenging endeavour.

We are glad to present these three Editorials to our EPS readers, because we are convinced that more research is needed, particularly on the most effective strategies for preventing and countering occupational stress and job burnout. A major problem in the work stress literature, as discussed by the three editorials, is that although work-related stressors have been identified, interventions have focused predominantly on individual factors (Edwards et al., 2003; Gilbody et al., 2006). These approaches, however, may be only partially effective, because recent research has demonstrated that organisational factors play a greater role in

I professionisti sanitari che si occupano di pazienti con patologie gravi e di lunga durata sono frequentemente esposti a sollecitazioni emotive stressanti e profondamente dolorose. Questi stati d'animo vanno dal bisogno di 'salvare' il paziente, al senso di fallimento e frustrazione quando la patologia progredisce, a sentimenti di impotenza nei confronti della malattia e conseguenti sentimenti di perdita, lutto, paura di ammalarsi, desiderio di rimanere emotivamente distanti dai pazienti, o di evitarli del tutto, per sfuggire a tali emozioni. Queste ultimo sono molto comuni nella pratica clinica quotidiana e spesso compromettono sia la qualità delle cure fornite, che il senso di benessere personale dei professionisti stessi (Meier et al., 2001). Tra gli operatori sanitari, coloro che lavorano in salute mentale appaiono essere i professionisti a più alto rischio di sviluppare stress lavorativo (Leary \& Brown, 1995; Nolan et al., 1995) e burnout (Leiter \& Harvie, 1996; Prosser et al., 1996; Thomsen et al., 1999).

Il burnout va tenuto concettualmente distinto dallo stress lavorativo: infatti, mentre quest'ultimo definisce un generico processo di adattamento temporaneo al lavoro accompagnato da sintomi psicologici e fisici, il primo rappresenta l'esito finale negativo di processo di adattamento nei confronti di una condizione di stress lavorativo prolungato. Il burnout, inoltre, si associa ad atteggiamenti e comportamenti autoprotettivi nei confronti dei destinatari delle cure, del lavoro in quanto tale e dell'organizzazione per la quale si lavora. Il burnout rappresenta una condizione di elevata occupational stress and job burnout than individual factors.

Moreover, several groups have called for multilevel theoretical models and research to explain the work-stress process (Bliese et al., 2002) and for a move beyond single phenomenon as explanations of work stress. Empirical recognition of the effects of worklife areas and contextual factors on occupational stress and burnout (Lasalvia et al., 2009) has expanded the range of options for organisational intervention. Although the potential value of organisational intervention is tremendous, these programmes are not easy to implement, because they frequently require complex collaboration and a considerable economic investment. Moreover, further research in mental health is needed to identify its problematic aspects, including the identification of variables influencing inappropriate termination of care by severely mentally ill with community-based psychiatric patients (Rossi et al., 2002), which might implicate them as targets for intervention. Lastly, additional research is necessary to determine the more effective forms of intervention for specific professional categories in mental health and the circumstances under which they work best.

criticità per l'assistenza psichiatrica, in quanto riduce le performance lavorative del personale e, conseguentemente, produce esiti clinici peggiori nei pazienti (Priebe et al., 2004). I servizi di salute mentale di molti paesi europei devono fare i conti con i tagli alle risorse finanziarie a loro destinate, con conseguenti ricadute occupazionali negative riguardo al numero di professionisti impiegati nel settore. Il problema è particolarmente sentito in quei Paesi dotati di un sistema sanitario nazionale, quali ad esempio la Gran Bretagna (Sainsbury Centre for Mental Health, 2001) e l'Italia (Lasalvia et al., 2007). In contesti di questo tipo, sintomi da stress lavorativo e burnout contribuiscono di fatto a depauperare le già scarse risorse umane in dotazione ai servizi.

Storicamente il burnout è stato considerato più come un problema del singolo che dell'organizzazione (Maslach \& Leiter, 1997). Gli studi sui determinanti del burnout nei servizi di salute mentale hanno in genere appuntato la propria attenzione su fattori individuali di tipo socio-demografico (Fagin et al., 1996; Edwards et al., 2000), su caratteristiche individuali di tipo lavorativo (Prosser et al., 1999; Kilfedder et al., 2001; Evans et al., 2006) o su tratti personologici predisponenti (Naisberg-Fennig et al., 1991; Deary et al., 1996a). Tuttavia, studi più recenti hanno ampliato la cornice teorica del concetto di burnout dal singolo lavoratore alla struttura organizzativa per la quale il singolo lavora. In tale ottica, Maslach \& Leiter (1997) hanno elaborato un nuovo modello interpretativo che 
pone al centro dell'attenzione il grado di adattamento/ disadattamento tra persona e lavoro. Tali discrepanze sono da considerarsi come i più importanti antecedenti del burnout e sono sperimentabili in sei ambiti della vita organizzativa (carico di lavoro, controllo, ricompense, senso comunitario, equità, valori). Leiter \& Maslach (2000) hanno conseguentemente proposto il cosiddetto 'Modello di Mediazione', secondo cui la sindrome del burnout ha maggiori probabilità di svilupparsi quando è presente una forte discordanza tra la natura del lavoro e la natura delle persone che svolgono tale lavoro. Questo modello offre un approccio molto promettente sia per la ricerca che per gli interventi applicativi, dal momento che si focalizza sul rapporto che lega il burnout ai fattori contestuali dell'ambiente lavorativo (Leiter \& Maslach, 2004). In questa prospettiva, esso può essere visto come una condizione di sofferenza individuale mediata dal contesto lavorativo, in cui i fattori organizzativi esercitano una maggiore influenza rispetto a quelli personali (Maslach \& Leiter, 2008).

Una recente indagine su larga scala condotta in Italia sugli operatori della salute mentale (Lasalvia et al., 2009), e che ha adottato la cornice di riferimento del 'Modello di Mediazione' di Leiter \& Maslach (2000), ha rilevato che i fattori organizzativi rappresentano gli unici predittori significativi delle tre dimensioni attraverso cui si articola il concetto di burnout (esaurimento emotivo, disaffezione lavorativa e inefficacia professionale). Inoltre, ciascuna dimensione del burnout riconosceva un set specifico di predittori di tipo organizzativo, in grado di esercitare il proprio effetto sui livelli di disagio sperimentati degli operatori, indipendentemente dal profilo professionale di appartenenza. Per quanto riguarda la forza dell'associazione tra variabili, un elevato carico di lavoro percepito rappresenta il predittore principale dell'esaurimento emotivo, scarsi livelli di riconoscimento e pochi cambiamenti positivi all'interno dell'organizzazione rappresentano i predittori principali della disaffezione lavorativa, la mancanza di partecipazione nei processi decisionali è il principale predittore del senso di inefficacia lavorativa. Questi risultati sembrano confermare empiricamente la validità del modello 'Job Demand-Resources' (JD-R), in base al quale le richieste provenienti dal contesto lavorativo e le risorse su cu i lavoratori possono fare affidamento rappresentano gli antecedenti più importanti, rispettivamente, della componente energia/esaurimento e disaffezione lavorativa del burnout (Bakker \& Demerouti, 2007). Il modello JD-R ipotizza, infatti, che il burnout sia determinato da due processi sottostanti: a) il senso di deplezione energetica, che è determinato dalle richieste eccessivamente elevate cui il lavoratore è sottoposto (es., pressioni relative ai tempi di realizzazione di un determinato compito, sollecitazioni emotive, compiti cognitivamente impegnativi, lavoro a turni) ed è associato alla componente esaurimento emotivo; b) l'erosione della motivazione, che è determinata dalla carenza di risorse presenti nel contesto lavorativo (es., supporto da parte dei supervisori, feed-back sul lavoro svolto, controllo sul processo lavorativo, varietà di mansioni da svolgere, incentivi economici) ed è associata con le componenti disaffezione lavorativa (cinismo) e inefficacia professionale.

Questo numero di Epidemiology and Psychiatric Sciences pubblica tre Editoriali che affrontano il delicato tema dello stress lavorativo e del burnout tra gli operatori della salute mentale. Abbiamo invitato tre noti esperti internazionali del settore che sono attivamente coinvolti anche nella ricerca empirica sul campo.

Dollard \& McTernan (2011) inquadrano il problema da un punto di vista generale e propongono un nuovo costrutto, il Clima di Sicurezza Psicosociale (Psychosocial Safety Climate, PSC), che può aiutare a comprendere meglio le dinamiche interpersonali e psicologiche che hanno luogo all'interno delle organizzazioni lavorative. Il PSC è definito come la percezione condivisa da parte dei lavoratori della presenza di un insieme di politiche, pratiche e procedure organizzative messe in atto a beneficio della loro salute e sicurezza psicologica, promosse in larga misura dal management dell'organizzazione stessa. Tale concetto si basa sull'assunto che il PSC deriva principalmente dalla priorità che la leadership di una data organizzazione conferisce al benessere psicologico dei lavoratori piuttosto che alla produttività: laddove esiste una precisa volontà politica in tal senso e il management persegue l'obiettivo di favorire l'equilibrio tra benessere dei lavoratori e produttività, ci si attende che siano presenti politiche, prassi e procedure che riflettano tale priorità (Dollard \& Karasek, 2010). Sulla base di questo principio, le condizioni lavorative (cioè, le richieste e le risorse) sono una conseguenza delle iniziative della leadership dell'organizzazione. In tal modo la teoria del PSC estende il modello 'Job Demands-Resources' (JD-R), nel senso che il PSC può essere considerato un precursore o antecedente delle condizioni lavorative (richieste e risorse) e non una conseguenza o un mediatore delle stesse. Studi recenti hanno dimostrato che il PSC è chiaramente un antecedente del clima che permea un determinato contesto lavorativo ed è associato al benessere psicologico attraverso il suo rapporto con l'ambiente di lavoro (Bond et al., 2010; Dollard \& Bakker, 2010). Infatti, il PSC è correlato negativamente con le richieste lavorative e positivamente con le risorse. Le richieste, a loro volta, sono predittori di burnout (esaurimento emotivo e disaffezione lavorativa), mentre le risorse sono predittori dell'impegno 
nei riguardi del lavoro. Sia il burnout che l'impegno sono associate (con segno evidentemente opposto) alla performance lavorativa. Pertanto, il PSC influenza la performance lavorativa o attraverso la compromissione dello stato di salute (cioè il burnout), o attraverso i processi motivazionali (cioè l'impegno nel lavoro). In considerazione delle caratteristiche del PSC, un'organizzazione che pone particolare attenzione al benessere e al senso di sicurezza psicologica dei propri dipendenti effettuerà richieste lavorative motivanti e che promuovono la salute (Dollard \& Bakker, 2010). Il PSC rappresenta pertanto un indicatore chiave dei rischi psicosociali legati all'ambiente lavorativo (elevate domande, scarse risorse), del benessere emotivo e dell'impegno dei dipendenti e un potenziale fattore di moderazione degli esiti connessi ai rischi psicosociali. Pertanto, il PSC può essere visto come un efficiente bersaglio per migliorare le condizioni lavorative, e conseguentemente, ridurre il burnout e incrementare impegno e produttività.

Tra gli operatori della salute mentale, gli psichiatri in particolare sembrano essere ad elevato rischio di sviluppare depressione, ansia, disturbi psichiatrici in genere e burnout (Naisberg-Fennig et al., 1991; Deary et al., 1996b; Thomsen et al., 1999; Lasalvia et al., 2009). Per questo motivo Kumar (2011) nel secondo editoriale focalizza la propria attenzione sulle caratteristiche dello stress lavorativo e del burnout negli psichiatri. L'articolo passa in primo luogo in rassegna le possibili ragioni che rendono tale professione così stressante, ponendo particolare enfasi, ovviamente, ai fattori di stress occupazionali. Tali fattori comprendono l'aggressività di alcuni pazienti, il suicidio cui vanno talora incontro i pazienti, la limitazione delle risorse, i reparti di degenza affollati, i cambiamenti culturali all'interno dei servizi di salute mentale, le elevate richieste lavorative, la scarsa definizione di ruolo, la responsabilità senza autorità, l'incapacità di realizzare modifiche sistemiche, conflitti tra la responsabilità nei confronti dello staff e quella nei confronti dei pazienti, l'isolamento professionale. Vengono poi analizzati gli specifici meccanismi che determinano il burnout tra gli psichiatri e discusse le conseguenze negative sulla professione dovute all'esposizione cronica a fattori stressanti di tipo lavorativo. Infine, pur riconoscendo il fatto che molti passi avanti sono stati compiuti nella comprensione dei fattori associati allo stress e al burnout negli psichiatri, Kumar (2011) sottolinea la necessità di approfondire le conoscenze sulle variabili in grado di generare un clima positivo negli ambienti di lavoro e che siano protettive rispetto allo stress lavorativo, invocando altresì il bisogno di implementare ricerche mirate sui fattori che consentano agli psichiatri di trarre soddisfazione e senso di realizzazione dal proprio lavoro.
Nel terzo editoriale, Priebe \& Reininghaus (2011) mettono in discussione il tradizionale paradigma di ricerca sullo stress occupazionale e il burnout, sollecitando il supena memto del modello negativo incentrato sullo stress a favore di un approccio che valorizzi gli aspetti positivi del lavoro: quei fattori, cioè, che promuovono 'l'impegno lavorativo', vale a dire una attitudine rispetto al lavoro che sia positiva, appagante e che consenta di svolgere il proprio compito in maniera motivata ed efficace; tale concezione è evidentemente agli antipodi rispetto al concetto di burnout (Bakker et al., 2008). La cornice generale di questo cambio di paradigma è dato dalla 'psicologica positiva' (Seligman \& Csikszentmihalyi, 2000), un movimento di ricerca empirica ed applicativa che enfatizza specificamente quegli aspetti della condizione umana legati alla felicità e al senso di autorealizzazione, allo scopo di definire $\mathrm{i}$ fattori in grado di determinare una buona riuscita nella vita (piuttosto che indirizzare gli sforzi per prevenire le situazioni negative) (Caprara et al., 2010).

In questo contesto, il concetto di 'impegno' sembra in grado dare conto di quegli aspetti che contribuiscono a promuovere un atteggiamento positivo nei confronti del lavoro e a determinare un clima di benessere organizzativo. Il lavoro in salute mentale pone generalmente gli operatori di fronte ad un'ampia gamma di esperienze, relazioni ed incontri, che sono talora a rischio di frustrazione e delusione, ma che possono anche generare entusiasmo, passione e soddisfazione; non deve pertanto sorprendere se gli operatori della salute mentale indichino la possibilità di stabilire una relazione positiva con i pazienti ed il senso di sfida quotidiana legato al lavoro come gli aspetti più appaganti della propria attività (Prosser et al., 1996; Galeazzi et al., 2004). Gli autori, pertanto, sottolineano la necessità di inaugurare una nuova linea di ricerca in grado di fornire maggiore comprensione sugli specifici meccanismi premianti che caratterizzano il lavoro in salute mentale e sui fattori che rendono tale attività un'affascinante e stimolante sfida.

Siamo lieti di presentare questi tre editoriali ai nostri lettori di EPS, in quanto siamo convinti che sia necessario promuovere più ricerca in questo settore, ed in particolare sulle strategie più efficaci per prevenire e contrastare lo stress lavorativo e il burnout. Uno dei problemi principali messi in luce dalla letteratura sullo stress lavorativo, come unanimemente sottolineato in tutti e tre gli editoriali, è che mentre la ricerca ha oramai identificato numerosi fattori stressogeni di tipo organizzativo, gli interventi esistenti tendono prevalentemente a concentrarsi sui fattori individuali (Edwards et al., 2003; Gilbody et al., 2006). Tali approcci, possono rivelarsi solo parzialmente efficaci, in quanto la ricerca più recente ha riscontrato che $\mathrm{i}$ 
fattori organizzativi giocano un ruolo di maggiore rilievo rispetto a quelli individuali nel determinare stress lavorativo e burnout. Inoltre, numerosi autori hanno proposto l'adozione di modelli teorici e approcci di ricerca di tipo multilevel per analizzare i processi alla base dello stress lavorativo (Bliese et al., 2002), suggerendo di andare oltre l'analisi delle caratteristiche individuali. Il riscontro empirico del ruolo giocato dalle aree della vita lavorativa e dei fattori contestuali sullo stress lavorativo ed il burnout (Lasalvia et al., 2009) ha ampliato la possibile gamma di opzioni di intervento a livello organizzativo. Sebbene sia potenzialmente enorme il valore degli interventi di tipo organizzativo, essi non sono sempre facili da implementare, in quanto richiedono un complesso grado di collaborazione e considerevoli investimenti economici. È necessario, inoltre, approfondire la ricerca nell'ambito delle organizzazioni che operano nel campo della salute mentale allo scopo di individuare gli aspetti problematici specifici relativi a questo settore, compresa l'identificazione delle variabili che influenzano l'interruzione non concordata dei rapporti con i servizi di comunità da parte dei pazienti con gravi disturbi mentali (Rossi et al., 2002), e che possono a loro volta essere oggetto di interventi mirati. Sarebbe, infine, importante che la ricerca riesca a definire quali specifiche forme di intervento Jono più adatte alle singole categorie professionali che operano nel campo della salute mentale e in quali specifiche circostanze possono risultare maggiormente efficaci.

\section{References}

Bakker AB, Demerouti E (2007). The job demands-resources model: state of the art. Journal of Managerial Psychology 22, 309-328.

Bakker A, Schaufeli W, Leiter M, Taris T (2008). Work engagement: an emerging concept in occupational health psychology. Work and Stress 22, 187-200.

Bliese PD, Jex SM, Halverson RR (2002). Integrating multilevel analyses and occupational stress theory. In Research in Occupational Stress and Well Being (ed. P. L. Perrewe and D. C. Ganster), pp. 217-259. JAI Press: Amsterdam

Bond S, Tuckey M, Dollard MF (2010). Psychosocial safety climate, workplace bullying and symptoms of post traumatic stress. Organization Development Journal 28, 37-56.

Caprara GV, Steca P, Alessandri G, Abela JR, McWhinnie CM (2010). Positive orientation: explorations on what is common to life satisfaction, self-esteem, and optimism. Epidemiologia e Psichiatria Sociale 19, 63-71.

Deary IJ, Agius RM, Sadler A (1996a). Personality and stress in consultant psychiatrists. International Journal of Social Psychiatry 42, 112-123.
Deary IJ, Blenkin H, Agius RM, Endler NS, Zealley H, Wood R (1996b). Models of job-related stress and personal achievement among consultant doctors. British Journal of Psychology 87, 3-29.

Dollard MF, Bakker AB (2010). Psychosocial safety climate as a precursor to conducive work environments, psychological health problems, and employee engagement. Journal of Occupational and Organizational Psychology 83, 579-599.

Dollard MF, Karasek R (2010). Building psychosocial safety climate: Evaluation of a socially coordinated PAR risk management stress prevention study. In Contemporary Occupational Health Psychology: Global Perspectives on Research and Practice (ed. J. Houdmont and S. Leka), pp. 208-234. Wiley-Blackwell: Chichester.

Dollard MF, McTernan WP (2011). Psychosocial safety climate: a multilevel theory of work stress in the health and community service sector. Epidemiology and Psychiatric Sciences, (in press) 2011.

Edwards D, Burnard P, Coyle D, Fothergill A, Hannigan B (2000). Stress and burnout in community mental health nursing: a review of the literature. Journal of Psychiatric and Mental Health Nursing 7, 7-14.

Edwards D, Burnard P, Owen M, Hannigan B, Fothergill A, Coyle D (2003). A systematic review of the effectiveness of stress-management interventions for mental health professionals. Journal of Psychiatric and Mental Health Nursing 10, 370-371.

Evans S, Huxley P, Gately C, Webber M, Mears A, Pajak S, Medina J, Kendall T, Katona C (2006). Mental health, burnout and job satisfaction among mental health social workers in England and Wales. British Journal of Psychiatry 188, 75-80.

Fagin L, Carson J, Leary J, De Villiers N, Bartlett H, O'Malley P, West M, Mc Elfatrick S, Brown D (1996). Stress, coping and burnout in mental nurses: findings from three research studies. International Journal of Social Psychiatry 42, 102-111.

Galeazzi G, Delmonte S, Fakhoury W, Priebe S (2004). Morale of mental health professionals in community mental health services of a Northern Italian province. Epidemiologia e Psichiatria Sociale 13, 191-197.

Gilbody S, Cahill J, Barkham M, Richards D, Bee P, Glanville J (2006). Can we improve the morale of staff working in psychiatric units? A systematic review. Journal of Mental Health 15, 7-17.

Kilfedder CJ, Power KG, Wells TJ (2001). Burnout in psychiatric nursing. Journal of Advanced Nursing 34, 383-96.

Kumar S (2011). Burnout and psychiatrists: what do we know and where to from here? Epidemiology and Psychiatric Sciences, (in press) 2011.

Lasalvia A, Bonetto C, Bertani M, Bissoli S, Cristofalo D, Marrella G, Ceccato E, Cremonese C, De Rossi M, Lazzarotto L, Marangon V, Morandin I, Zucchetto M, Tansella M, Ruggeri M (2009). Influence of perceived organisational factors on job burnout: survey of community mental health staff. British Journal of Psychiatry 195, 537-544.

Lasalvia A, Gentile B, Ruggeri M, Marcolin A, Nosè F, Cappellari L, Lamonaca D, Toniolo E, Busana C, Campedelli A, Cuccato G, Danieli A, De Nardi F, De 
Nardo V, Destro E, Favaretto G, Frazzingaro S, Giacopuzzi M, Pristinger P, Pullia G, Rodighiero S, Tito P, Aprile F, Nicolaou S, Coppola G, Garzotto N, Gottardi U, Lazzarin E, Migliorini G, Pavan L, Ramaciotti F, Roveroni P, Russo S, Urbani P, Tansella M; Gruppo Picos-Veneto (2007). Heterogeneity of the Departments of Mental Health in the Veneto Region ten years after the National Plan 1994-96 for Mental Health. Which implication for clinical practice? Findings from the PICOS Project. Epidemiologia e Psychiatria Sociale 16, 59-70.

Leary J, Brown D (1995). Findings from the Claybury study for ward based psychiatric nurses and comparisons with community psychiatric nurses. In Stress and Coping in Mental Health Nursing (ed. J. Carson, L. Fagin and S. Ritter), pp. 87-114. Chapman and Hall: London.

Leiter MP, Harvie PL (1996). Burnout among mental health workers: a review and a research agenda. International Journal of Social Psychiatry 42, 90-101.

Leiter MP, Maslach C (2000). Preventing Burnout and Building Engagement: A Complete Program for Organizational Renewal. Jossey-Bass: San Francisco.

Leiter MP, Maslach C (2004). Areas of worklife: A structured approach to organizational predictors of job burnout. In Research in Occupational Stress and Well Being:

Vol. 3. Emotional and Physiological Processes and Positive Intervention Strategies (ed. P. L. Perrewe and D. C. Ganster), pp. 91-134. JAI Press/Elsevier: Oxford.

Maslach C, Leiter MP (1997). The truth about burnout. How organizations cause personal stress and what to do about it. Jossey-Bass: San Francisco, CA.

Maslach C, Leiter MP (2008). Early predictors of job burnout and engagement. Journal of Applied Psychology 98, 498-512.

Meier DE, Back AL, Morrison RS (2001). The inner life of physicians and care of the seriously ill. Journal of the American Medical Association 286, 3007-3014.

Naisberg-Fennig S, Fennig S, Keinan G, Elizur A (1991). Personality characteristics and proneness to burnout: a study among psychiatrists. Stress Medicine 7, 201-205.
Nolan P, Cushway D, Tyler P (1995). A measurement tool for assessing stress among mental health nurses. Nursing Standard 9, 36-39.

Priebe S, Fakhoury W, White I, Watts J, Bebbington P, Billings J, Burns T, Johnson S, Muijen M, Ryrie I, Wright C \& Pan-London Assertive Outreach Study Group (2004). Characteristics of teams, staff and patients: associations with outcomes of patients in assertive outreach. British Journal of Psychiatry 185, 306-311.

Priebe S, Reininghaus U (2011). Fired up, not burnt out focusing on the rewards of working in psychiatry. Epidemiology and Psychiatric Sciences, doi:10.1017/ S2045796011000606, published online by Cambridge University Press 19 August 2011.

Prosser D, Johnson S, Kuipers E, Szmukler G, Bebbington P, Thornicroft G (1996). Mental health, "burnout" and job satisfaction among hospital and community-based mental health staff. British Journal of Psychiatry 169, 334-337.

Prosser D, Johnson S, Kuipers E, Dunn G, Szmukler G, Reid Y, Bebbington P, Thornicroft G (1999). Mental health, "burnout" and job satisfaction in a longitudinal study of mental health staff. Social Psychiatric Epidemiology 34, 295-300.

Rossi A, Amaddeo F, Bisoffi G, Ruggeri M, Thornicroft G, Tansella M (2002). Dropping out of care: inappropriate terminations of contact with community-based psychiatric services. British Journal of Psychiatry 181, 331-338.

Sainsbury Centre for Mental Health (2001). Finding and Keeping: A Review of Recruitment and Retention in and Keeping: A Review Recruitment and Retention in the Mental Health Workforce. The Sainsbury Centre: London.

Seligman M, Csikszentmihalyi M (2000). Positive psychology: an introduction. American Psychologist 55, 5-14.

Thomsen S, Soares J, Nolan P, Dallender J, Arnetz B (1999). Feelings of professional fulfilment and exhaustion in mental health personnel: the importance of organisational and individual factors. Psychotherapy and Psychosomatics 68, 157-164. 